Article

\title{
Research on Absolute Calibration of GNSS Receiver Delay through Clock-Steering Characterization
}

\author{
Feng Zhu 1,2,3,*(D), Huijun Zhang 1,2,3, Luxi Huang 1,2,3, Xiaohui Li 1,2,3 and Ping Feng 1,2,3 \\ 1 National Time Service Center, Chinese Academy of Science, Xi'an 710600, China; zhj@ntsc.ac.cn (H.Z.); \\ huangluxi@ntsc.ac.cn (L.H.); xiaohui@ntsc.ac.cn (X.L.); pingfp@ntsc.ac.cn (P.F.) \\ 2 Technology and Engineering Center for Space Utilization, University of Chinese Academy of Science, \\ Beijing 100039, China \\ 3 Key Laboratory of Precise Navigation and Timing Technology, Chinese Academy Science, \\ Xi'an 710600, China \\ * Correspondence: zhufeng@ntsc.ac.cn; Tel.: +86-29-8389-0447; Fax: +86-29-8389-0326
}

Received: 23 September 2020; Accepted: 22 October 2020; Published: 25 October 2020

\begin{abstract}
The receiver delay has a significant impact on global navigation satellite system (GNSS) time measurement. This article comprehensively analyzes the difficulty, composition, principle, and calculation of GNSS receiver delay. A universal method, based on clock-steering characterization, is proposed to absolutely calibrate all types of receivers. We use a hardware simulator to design several experiments to test the performance of GNSS receiver delay for different receiver types, radio frequency (RF) signals, operation status and time-to-phase (TtP). At first, through the receivers of Novatel and Septentrio, the channel delay of Septentrio is $2 \mathrm{~ns}$ far lower than $65 \mathrm{~ns}$ for Novatel, and for the inter-frequency bias of GLONASS L1, Septentrio tends to increase within $10 \mathrm{~ns}$ compared with decreasing of Novatel within $5 \mathrm{~ns}$. Secondly, a representative receiver of UniNav-BDS (BeiDou) is chosen to test the influence of Ttp which may be ignored by users. Under continuous operation, the receiver delay shows a monotone reduction of $10 \mathrm{~ns}$ as TtP increased by $10 \mathrm{~ns}$. However, under on-off operation, the receiver delay represents periodic variation. Through a zero-baseline comparison, we verifies the relation between receiver delay and TtP. At last, the article analyzes instrument errors and measurement errors in the experiment, and the combined uncertainty of absolute calibration is calculated with $1.36 \mathrm{~ns}$.
\end{abstract}

Keywords: receiver delay; absolute calibration; clock-steering; pulse-per-second (PPS); TtC (time-to-code); TtP (time-to-phase)

\section{Introduction}

The calibration of global navigation satellite systems (GNSS) receiver delay is always a difficult field in navigation [1]. Due to different characterizations of receivers, there are also differences in calibration methods, which should be studied with pertinence solutions [2,3]. At present the most widely used method is the relative calibration developed by Bureau International des Poids et Mesures (BIPM), with the advantages of simple operation and high precision, which is mainly used in timing transfer field such as GNSS common-view (CV) or two-way satellite time and frequency transfer (TWSTFT) $[4,5]$. However, this method can only obtain the relative delay between the calibrated receiver and the reference receiver [6]. In some applications such as one-way timing, timing evaluation, and time-offset monitoring, the receiver delay must be accurately obtained through absolute calibration $[7,8]$.

The absolute calibration method is first proposed by the Colorado university and put into operation by Naval Research Laboratory (NRL), which can absolutely calibrate GNSS receiver delay for different frequency band, code type, and signal modulation by using a GNSS hardware simulator [9-11]. In most 
experiments the receivers selected by NRL represented the external $10 \mathrm{MHz}$ and pulse-per-second (PPS) from the atomic clock. As the reference time fixed the receiver delay stably, the variation of calibration result is mostly focused on temperature sensitivity, reference clock, source bandwidth, and data processing [12-14]. Nevertheless, the phase difference between external $10 \mathrm{MHz}$ and PPS named time-to-phase (TtP) also affected the calibration result [15]. Although several related papers from NRL or Centre National d'Etudes Spatiales (CNES) had analyzed the relation between receiver delay and $\mathrm{TtP}$, there is still lack of comprehensive research on its principle and method $[9,16]$. Meanwhile, especially for the timing receivers without PPS input, hardly any papers had analyzed the calibration method, which should also be studied as key research.

In order to research the absolute calibration method of GNSS receivers delay, the article first analyzed the timing characterization of GNSS receiver delay. Through dividing the receiver delay into parts, we presented the method of absolute calibration based on the clock-steering characterization. Using a GNSS hardware simulator of Spirent GSS8000 to establish an absolute calibration platform. Two types of GNSS receivers like Novatel and Septentrio, both without PPS input, are calibrated for comparison. After self-calibration of the simulator, the experiment absolutely calibrated the channel delay of GPS L1/L2 and inter-frequency bias of GLONASS L1. Through comparing the calibration result of PPS output delay, we specially tested the clock-steering performance of receiver PPS output. As a representative receiver with external $10 \mathrm{MHz}$ and PPS, UniNav-BDS (BeiDou) is absolutely calibrated with the variation of TtP. A zero-baseline comparison verified the relation between calibration result and TtP under the case of continuous operation and on-off operation. Finally, we analyzed the calibration uncertainty, and the discussion is given.

\section{Timing Characterization of GNSS Receiver Delay}

As time equipment, each GNSS receiver must have a receiver time which is short for RecT, and especially for well-timing receivers, RecT has not only high stability but also keeps synchronous to a unified time scale [17]. After successful positioning, the receiver always represents RecT with PPS output for user timing, and most measurement values from GNSS receiver are generated under RecT drive. In order to comprehend the composition of receiver delay better, we analyze the working process of RecT.

\subsection{Clock-Steering Characterization}

RecT has been generated by the receiver clock such as an oscillator since the receiver starts operating. As the rising edge of RecT is random, the receiver will reduce the clock-bias by adjusting RecT to GNSS time (GNSST) or PPS input, which is named clock-steering processing as shown in Figure 1 [18].

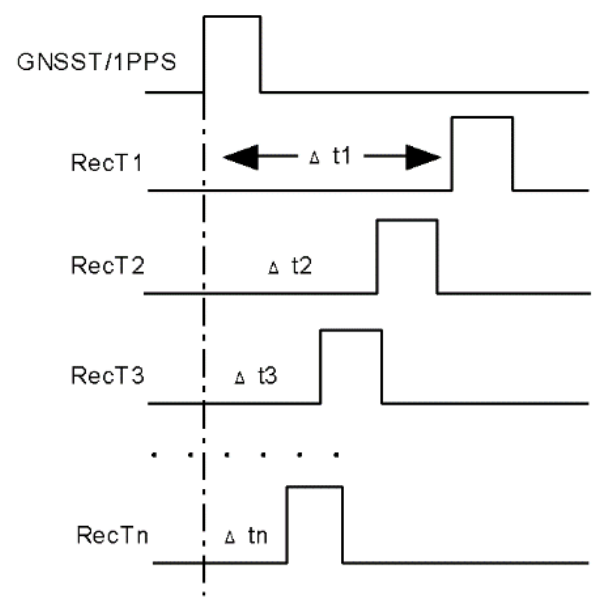

Figure 1. Clock-steering processing of the receiver time. 
At the beginning of receiver operation, $\operatorname{Rec} T$ is frequently divided into generation by the receiver clock at any time. In order to synchronize $\operatorname{RecT}$ with a stable time, the receiver will adjust $\operatorname{Rec} T_{1}$ by clock-bias $\Delta t_{1}$ after first positioning. If the receiver does not have PPS input, RecT will keep synchronous to global navigation satellite systems time (GNSST) such as GPS time (GPST), otherwise PPS input instead. Ideally $\Delta t_{n}$ is equal to zero as RecT tends to be stable. However, due to hardware configuration $\Delta t_{n}$ can only be accurate within a certain range, such as receiver clock of $100 \mathrm{MHz}$ only accurate to $10 \mathrm{~ns}$, which exists an unknown delay in pseudorange. For some receivers such as Septentrio $\Delta t_{n}$ has already been deducted from the original measurement, which means the pseudorange is a modified value via clock-bias correction.

\subsection{Composition of the Receiver Delay}

The receiver delay commonly refers to the time bias from radio frequency (RF) signal input to measurement data output. For the timing receiver, there is also PPS output which always synchronizes with the measurement data, in other words, the timing receiver delay can be defined as the time bias from RF signal input to PPS output $[10,19]$.

Altogether, GNSS receiver uses the clock-bias to adjust RecT after until clock-bias under the minimum range, which represents RecT with PPS output. Therefore, we divide GNSS receiver delay into two parts as shown in Figure 2. One is the channel delay existing in pseudorange, the other is PPS output delay caused by hardware link. Compared with the relatively fixed PPS output delay, the channel delay varies from different RF signals, reference clocks, parameter settings, etc.

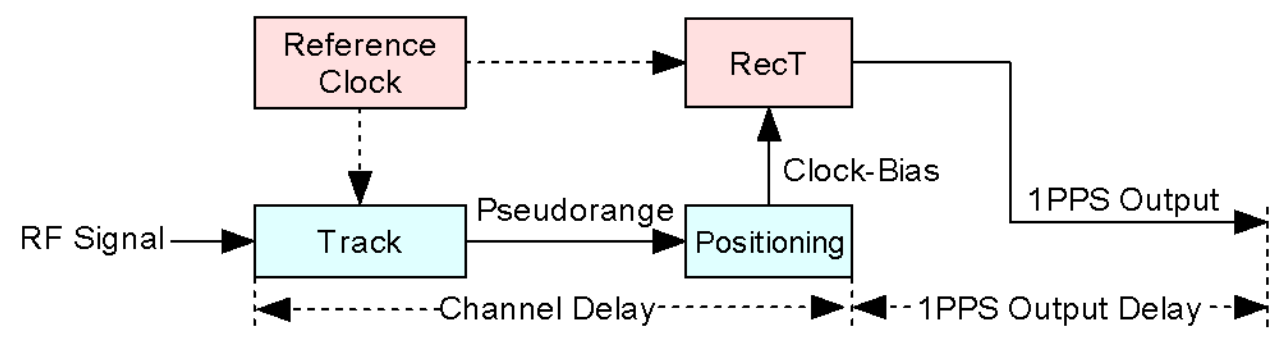

Figure 2. Composition of the receiver delay.

\section{Method of Absolute Calibration on GNSS Receiver Delay}

Using a GNSS hardware simulator instead of the real satellites, the principle of absolute calibration is shown in Figure 3. An atomic clock provides the simulator and receiver with the reference clock, and through sending PPS and RF signal to GNSS receiver, the simulator should be self-calibrated at first. A time interval counter measures the bias of PPS output between the receiver PPS(r) and simulator PPS(s). Therefore the data is collected by the computer.

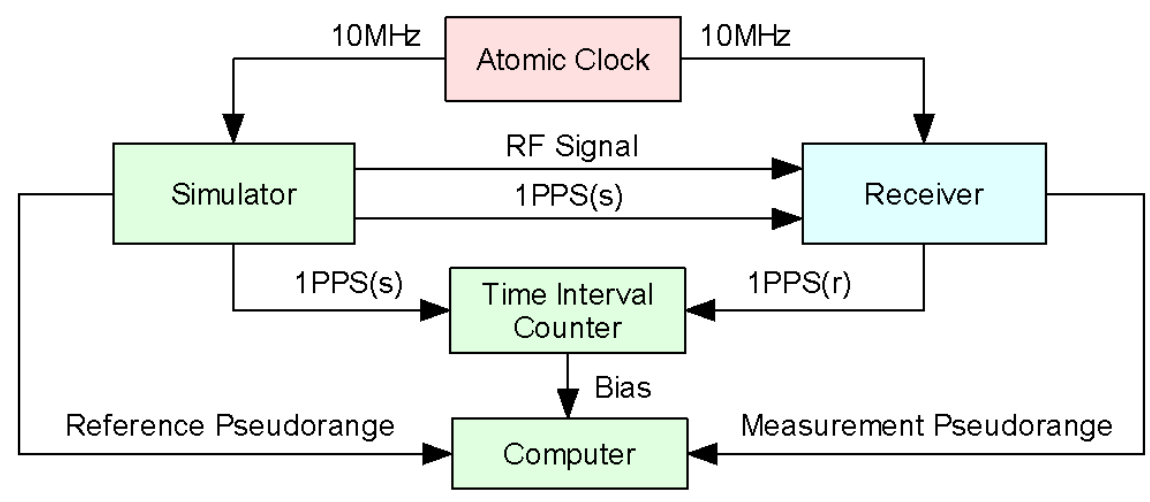

Figure 3. Principle of absolute calibration. 
In order to analyze the principle of absolute calibration better, we show the calculation process of the receiver delay as follows.

1. The reference pseudorange represents the ideal distance from the satellite to the receiver, which can be expressed by the formula as below:

$$
\rho_{s}=R+c \cdot\left(\tau_{\text {clk }}+\tau_{\text {iono }}+\tau_{\text {trop }}\right)
$$

where $\rho_{s}$ is the reference pseudorange from the simulator, $R$ is the geometry path from the satellite to the receiver, $\tau_{c l k}$ is the satellite clock-bias, $\tau_{i o n o}$ and $\tau_{\text {trop }}$ are relatively the ionosphere delay and troposphere delay.

$\rho_{S}$ is calculated by the simulator based on its system time like PPS(s), but it exists the simulator delay between RF signal and PPS(s), which is mainly caused by simulator channel and transmission cable.

2. The actual pseudorange reaching receiver includes the reference pseudorange and simulator delay:

$$
\rho_{s}{ }^{\prime}=\rho_{s}+c \cdot \tau_{\text {sim }}=R+c \cdot\left(\tau_{\text {clk }}+\tau_{\text {trop }}+\tau_{\text {iiono }}+\tau_{\text {sim }}\right)
$$

where $\rho_{s}{ }^{\prime}$ is the actual reference pseudorange, $\tau_{\text {sim }}$ is the simulator delay between zero-value of RF signal and PPS(s) named time-to-code (TtC), which can be measured from the code phase reversal point relative to rising edge of PPS by an oscilloscope [20].

3. The measurement pseudorange represents the actual distance of signal transmission process, which can be expressed by the formula as below:

$$
\rho_{r}=R+c \cdot\left(\tau_{\text {clk }}+\tau_{\text {trop }}+\tau_{\text {iono }}+\Delta t+\tau_{\text {rev }}\right)+R_{m p}+n
$$

where $\rho_{r}$ is the measurement pseudorange from receiver, $\Delta t$ is the receiver clock-bias, $\tau_{\text {rev }}$ is the receiver delay, $R_{m p}$ is the multipath interference, and $n$ is the measurement noise. Considering RF signal directly reaching the receiver via cable instead of an antenna, $R_{m p}$ can be ignored in the condition of impedance matching [21].

4. As the simulator can avoid ephemeris error, clock error, and atmosphere error, according to the Equations (2) and (3), the receiver delay can be calculated by the formula as below:

$$
\tau_{\text {rev }}=\left(\rho_{r}-\rho_{s}\right) / c-\Delta t-\tau_{\text {sim }}+n
$$

$\tau_{\text {rev }}$ is derived from pseudorange observation equation, which only represents the channel delay. However, $\Delta t$ is calculated from positioning equation, which includes the channel delay. As the true clock-bias from pseudorange cannot be deducted in reality, we consider it is part of channel delay. In other words, under the homologous common-clock condition, receiver clock-bias is the main factor of channel delay. Thus the channel delay can be expressed by the formula as below:

$$
\tau_{\text {chan }}=\left(\rho_{r}-\rho_{s}\right) / c-\tau_{\text {sim }}+n
$$

where $\tau_{\text {chan }}$ is the channel delay, $\rho_{s}$ is obtained from the simulator, $\rho_{r}$ is measured by the receiver, $\tau_{s i m}$ is calibrated by the oscilloscope. The inevitable $n$ can be processed by data smoothing. For PPS output delay, the measurement of time interval counter should be added into the calculation.

5. PPS output delay mainly refers to the link delay from internal generation to hardware port, and Figure 4 is shown for a better description: 


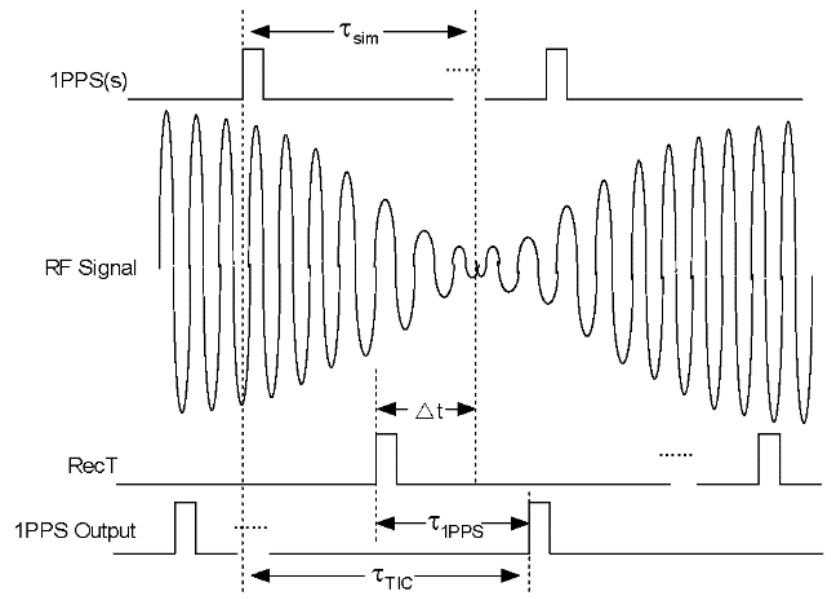

Figure 4. Calibration method of pulse-per-second (PPS) output delay.

$\tau_{T I C}$ measured from time interval counter represents the bias between PPS(s) and PPS(r), and $\Delta t$ calculated from pseudorange observation equation represents the bias between zero-value of RF signal and RecT. If PPS output does not exist the link delay which means $\tau_{1 P P S}$ is zero, $\tau_{\text {TIC }}$ plus $\Delta t$ is equal to $\tau_{\text {sim }}$. However, PPS output delay results in inequality, $\tau_{1 P P S}$ can be subtracted from the formula as below:

$$
\tau_{1 P P S}=\tau_{T I C}+\Delta t-\tau_{\text {sim }}
$$

Here a precondition of RecT is leading relatively to the zero-value of RF signal. Otherwise minus should replace plus in front of $\Delta t$. Meanwhile, under different frequency band, code type, or signal modulation the receiver delay is also different, especially for the channel delay, which should be calibrated separately.

\section{Calibration Results}

\subsection{Simulator Self-Calibration}

The simulator (Spirent GSS8000) is set to open one channel of No.1 satellite with GEO status, and GPS L1 carrier is modulated by P-code without navigation message. As the maximum power of RF signal is about $-60 \mathrm{dBm}$, two amplifiers are added behind RF signal output to ensure the oscilloscope can acquire the true signal. The oscilloscope (Tek DPO 71604C) is captured as Figure 5a,b shows the total delay of amplifiers, cables and adapters is $12.47 \mathrm{~ns}$ measured by a vector network analyzer(Agilent PNA-X N5242A) [10].

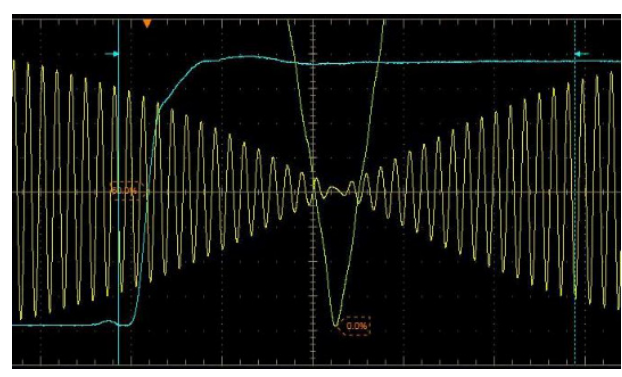

(a)

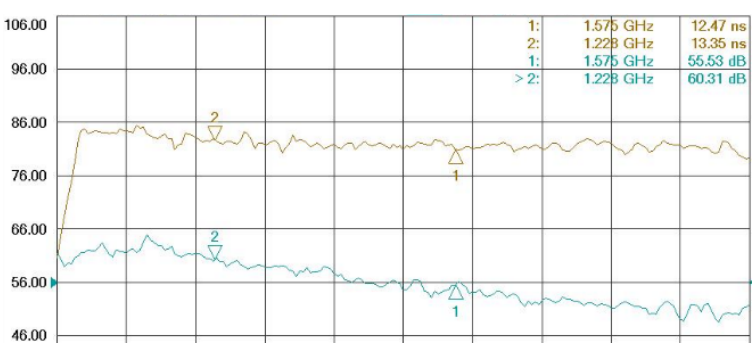

(b)

Figure 5. The calibration result of simulator: (a) time-to-code ( $\mathrm{TtC}$ ) measurement by the oscilloscope; (b) amplifiers delay measurement by the vector network analyzer. 
In order to directly find the code phase reversal point of RF signal, Hilbert transform is added into the oscilloscope. The 50\% level of PPS rising edge is referred as the trigger, and the measurement of $\mathrm{TtC}$ is $8.24 \mathrm{~ns}$.

An attenuator with delay 3.89 ns obtained from simulator product documentation should also be considered into calibration, which is added in the back RF port with low power for receiver calibration, as the above process used the front RF port with high power for simulator calibration.

$$
\tau_{\text {sim }}=\mathrm{TtC}+\tau_{\text {Attenuator }}-\tau_{\text {Amplifier }}=8.24 \mathrm{~ns}+3.89 \mathrm{~ns}-12.47 \mathrm{~ns}=-0.34 \mathrm{~ns}
$$

Thus, the calibration result of GPS L1 is -0.34 ns as above, and in this way, the results of other GNSS signal can be self-calibrated as well.

\subsection{Calibration of Receiver Delay}

\subsubsection{Channel Delay of GPS L1/L2}

The $10 \mathrm{MHz}$ signal is used as the external reference for the simulator and the receivers of Novatel OEMV-3G and Septentrio Polarx4 Pro. We set each receiver channel to track the specified satellite. The channel delay under GPS L1/L2 is shown as Figure 6, with the abscissa of testing time and ordinate of channel delay.

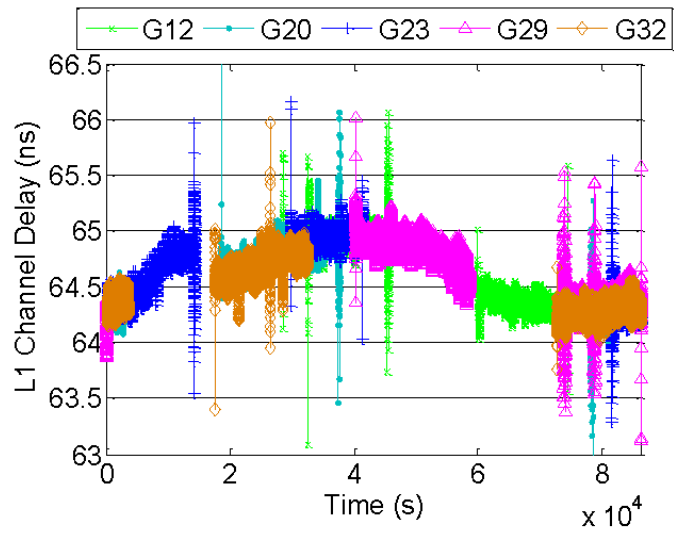

(a)

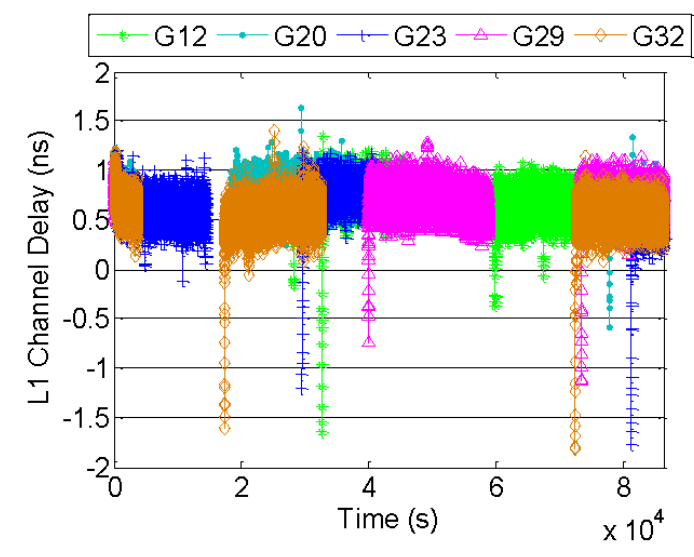

(c)

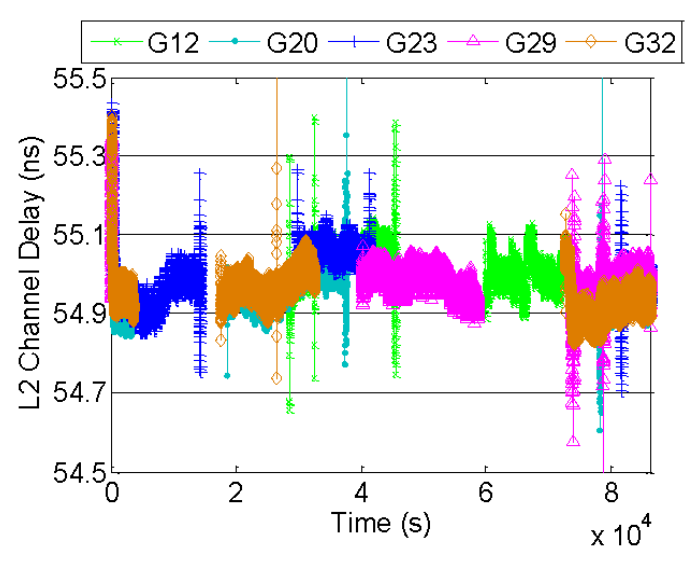

(b)

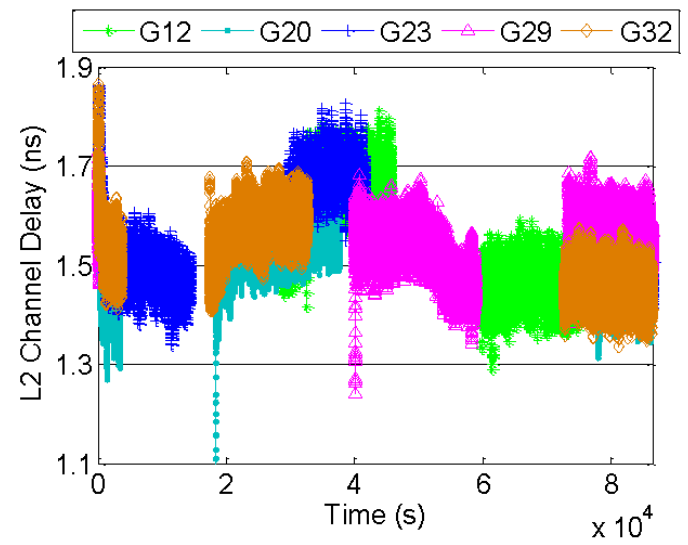

(d)

Figure 6. The calibration results of channel delay: (a) Novatel-GPS L1; (b) Novatel-GPS L2; (c) Septentrio-GPS L1; (d) Septentrio-GPS L2. 
Five satellites results are selected to calculate the channel delay of each receiver. Although sometimes the channel delay fluctuated about $2 \mathrm{~ns}$, the relative bias among different channels is nearly under $0.2 \mathrm{~ns}$, which can be ignored in the calibration. Taking the average of channel delay as a result, the data statistics are shown in Table 1.

Table 1. Channel delay statistics under GPS L1/L2 (unit: ns).

\begin{tabular}{ccccc}
\hline \multirow{2}{*}{ Channel } & \multicolumn{2}{c}{ Novatel } & \multicolumn{2}{c}{ Septentrio } \\
\cline { 2 - 5 } & L1 & L2 & L1 & L2 \\
\hline 1 & 64.67 & 55.03 & 0.72 & 1.56 \\
2 & 64.61 & 54.95 & 0.74 & 1.50 \\
3 & 64.68 & 55.00 & 0.67 & 1.57 \\
4 & 64.63 & 54.98 & 0.70 & 1.55 \\
5 & 64.49 & 54.95 & 0.56 & 1.52 \\
\hline
\end{tabular}

By contrast, the channel delay of Septentrio is under 2 ns far lower than 65 ns for Novatel, because the pseudorange from Septentrio has deducted $\Delta t$ via clock-bias correction. Meanwhile, the inter-frequency bias between L1 and L2 makes a great difference, $10 \mathrm{~ns}$ for Novatel and 1 ns for Septentrio, which is mainly due to hardware link and filter group delay.

\subsubsection{Inter-Frequency Bias of GLONASS L1}

From the last section, it can be seen an inter-frequency bias between GPS L1 and L2. As GLONASS uses frequency division multiple access (FDMA) technique to transmit signal, it is necessary to calibrate the inter-frequency bias among receiver channels under GLONASS L1 [2,22].

$$
f_{L 1}^{K}[H z]=1602.10^{6}+K \cdot 562500
$$

GLONASS signal has 14 carrier frequencies as the frequency number $\mathrm{K}$ variable, and all the satellites launched after 2005 use K(-7 to 6). Ignoring the receiver channel bias under 0.2 ns, we set each channel of GLONASS L1 transmitted No.1 satellite at different K. The calibration results of two receivers are shown as Figure 7.

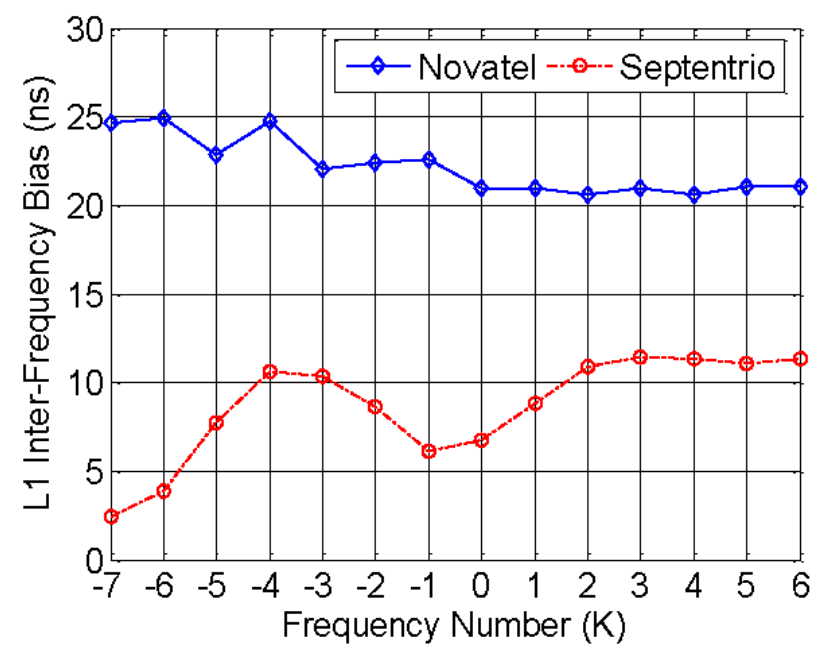

Figure 7. Inter-frequency bias of GLONASS L1.

The results between Novatel and Septentrio differ a lot. As $\mathrm{K}$ from -7 to 6 , the inter-frequency bias of Novatel is monotone decreasing within $5 \mathrm{~ns}$, but Septentrio is monotone increasing within $10 \mathrm{~ns}$. If taking -7 as the reference frequency number of GLONASS L1, the GPS/GLONASS time offset of the receiver is the inter-frequency bias at $\mathrm{K}=-7$, with $24.68 \mathrm{~ns}$ for Novatel and $6.78 \mathrm{~ns}$ for Septentrio. 


\subsubsection{PPS Output Delay}

After the receiver have operated stably, PPS output of the simulator and receiver are put into time interval counter of SR620, and the bias measured by SR620 is shown as Figure 8. The blue line with left ordinate represents PPS bias form SR620, and the red line with right ordinate represents clock-bias from receiver.

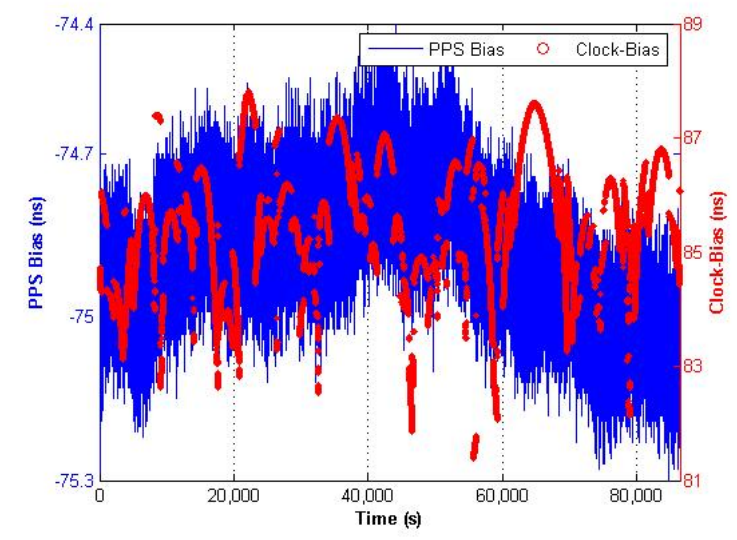

(a)

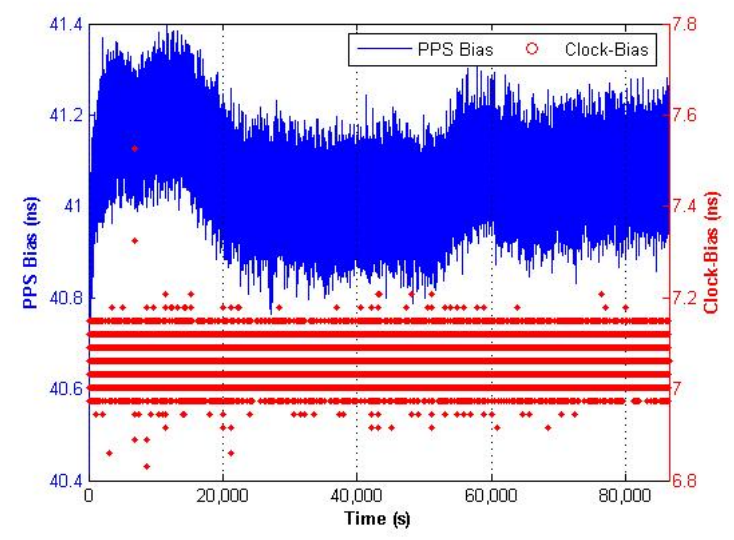

(b)

Figure 8. The calibration results of PPS output delay: (a) Novatel; (b) Septentrio.

As PPS output of simulator is the trigger signal in SR620, SR620 PPS bias of Novatel is minus, which meant receiver time of Novatel is leading relatively to GPST. Compared with Septentrio, the clock-bias of Novatel fluctuates larger than PPS bias, thus indicates the synchronous relation between RecT and PPS output may be different, which should be analyzed with pertinence test.

Taking the average of measurement as a result, the PPS output delay statistics are shown in Table 2, with $11.05 \mathrm{~ns}$ for Novatel and $48.46 \mathrm{~ns}$ for Septentrio.

Table 2. PPS output delay statistics (unit: ns).

\begin{tabular}{cccc}
\hline Receiver & PPS Bias & Clock-Bias & PPS Output Delay \\
\hline Novatel & -74.83 & 85.54 & 11.05 \\
Septentrio & 41.07 & 7.06 & 48.46 \\
\hline
\end{tabular}

\subsubsection{Test on the Clock-Steering Performance of Receiver PPS Output}

Many users consider PPS output of the receiver represents GNSST. However, due to different receiver hardware, operation model, and parameter setting, PPS output may not totally synchronize with GNSST. From the last section, PPS output of Novatel is different from Septentrio. By using three sets of SR620, we design an experiment to test the clock-steering performance of receiver PPS output. The $10 \mathrm{MHz}$ signal from the atomic clock of $10 \mathrm{MHz}(\mathrm{ref})$ is only used as the external reference for the receiver, and PPS signal from the atomic clock of PPS(ref) is individually put into three sets of SR620 as the trigger signal. PPS output from the receiver of PPS(r), $10 \mathrm{MHz}(\mathrm{ref})$ and PPS output from the simulator of PPS(s) are relatively put into SR620(1), SR620(2), and SR620(3). As the reference clock of the simulator is different from the receiver, we compare the bias of SR620(1) with SR620(2) and SR620(3) to test PPS output, with the method shown as Figure 9a. 


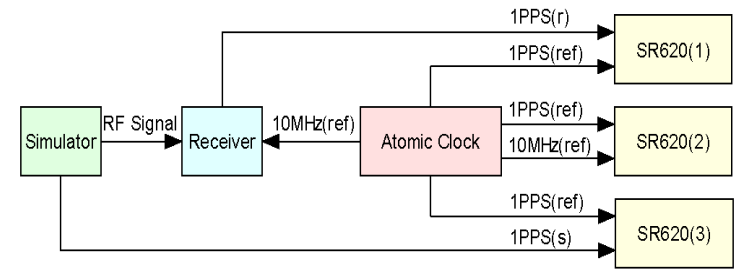

(a)

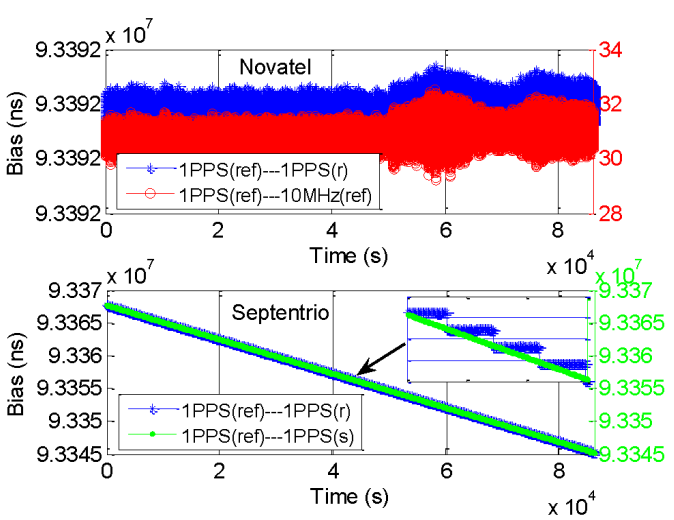

(b)

Figure 9. Testing of receiver PPS output: (a) testing method; (b) testing result, with the top panel of Novatel and the bottom panel of Septentrio.

Figure 9b shows SR620(1) bias of Novatel consistent with SR620(2), but SR620(1) bias of Septentrio consistent with SR620(3). Visibly, PPS(r) of Novatel or Septentrio is shown as the blue line steering to PPS(s). Along with the testing time PPS(r) of Septentrio synchronizes with PPS(s) shown as a green line in the bottom panel, but PPS(r) of Novatel synchronizes with $10 \mathrm{MHz}(\mathrm{ref})$ shown as red line in the top panel. Since PPS(s) from the simulator is defined as GPST, it indicates PPS output of Novatel is maintained by the reference clock after steering to GPST, but PPS output of Septentrio is steering to GPST all the while. From the watch window in the bottom panel, PPS output of Septentrio represents the ladder-like variation of $20 \mathrm{~ns}$, which reflects the synchronous precision of PPS output with $20 \mathrm{~ns}$.

\subsection{Influence of Ttp on Receiver Delay}

\subsubsection{Relation between $\mathrm{TtP}$ and RecT}

Both of the receivers chosen in above experiment do not have PPS input, which represents RecT from the atomic clock as the local reference. For other types of receivers, RecT will steer to the external PPS. Although the calibration method is the same as mentioned above, the calibration result especially for channel delay is affected with time-to-phase (TtP), which may be ignored by users [23].

The phase offset between external PPS input and $10 \mathrm{MHz}$ reference is named TtP, which is defined from the rising edge of PPS to zero-value of $10 \mathrm{MHz}$ shown as Figure 10a [24]. For few developed receivers, internal clock system has synchronized RecT with external PPS before operating like TtP $=0$, the receiver delay is not related with TtP. However, most of the commercial receivers do not manage TtP, which means RecT synchronizes with $10 \mathrm{MHz}$ reference firstly and steers to PPS input secondly. Consequently, RecT will correspond to change through TtP changing, shown as Figure 10b.

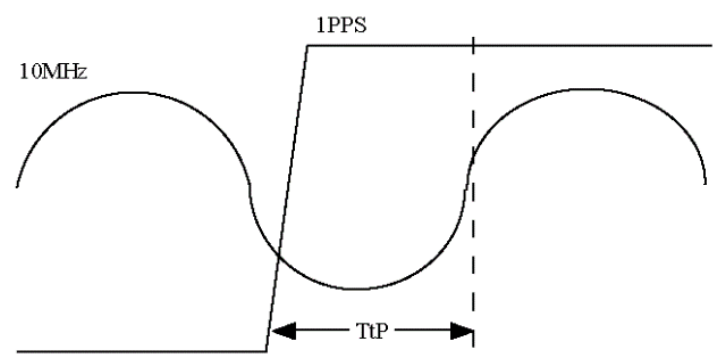

(a)

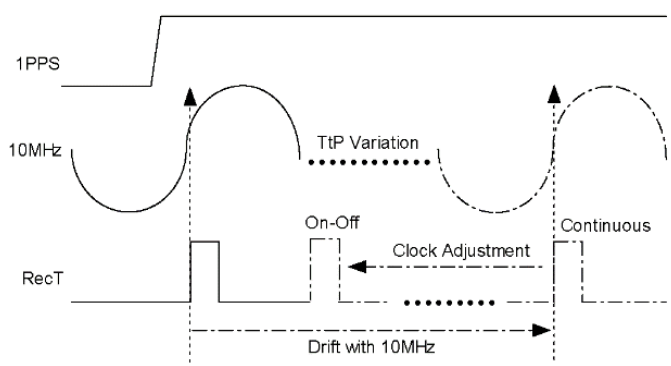

(b)

Figure 10. Relation between time-to-phase $(\mathrm{TtP})$ and receiver time $(\operatorname{RecT})$ : (a) definition of $\mathrm{TtP}$; (b) Ttp impact on RecT. 
While the change of $10 \mathrm{MHz}$ reference results in $\mathrm{TtP}$ variation, it represents RecT synchronous with the change of $10 \mathrm{MHz}$ phase. In the case of continuous operation, RecT is monotone changing as $\mathrm{TtP}$ variation, which does not adjust clock-bias after successful positioning. In the case of on-off operation, once the clock-bias exceeds adjustment threshold, the restarted receiver has to adjust clock-bias over again, which will keep RecT with the previous cycle of $10 \mathrm{MHz}$ phase until clock-bias less than adjustment threshold. Thus RecT is periodic changing as TtP changing.

Whichever continuous operation or on-off operation, the change of RecT will directly affect the measurement pseudorange from the receiver, which impacts on calibration result of channel delay.

\subsubsection{TtP Impact on Channel Delay}

A representative receiver of UniNav-BDS, developed by National University of Defence Technology China, is chosen in the calibration, which is used to test the variation of channel delay through TtP changing, shown as Figure 11a [25]. The principle is almost the same as above, and the only difference is that a phase offset generator of SDI hrog-10 is added in $10 \mathrm{MHz}$ reference, which is used to adjust the phase of the $10 \mathrm{MHz}$ signal. Under continuous operation and on-off operation, we adjust the phase of $10 \mathrm{MHz}$ reference with 10 ns every time, respectively acquiring the pseudorange bias between simulator and receiver to compare the variation of channel delay.

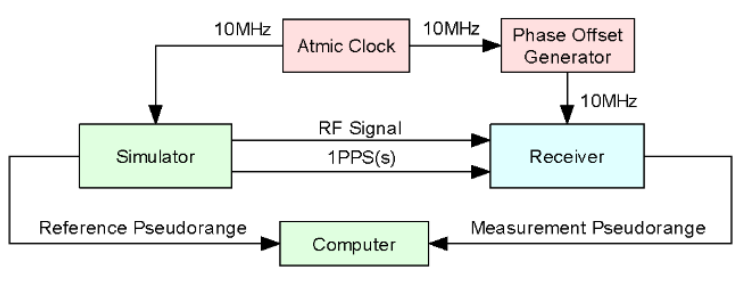

(a)

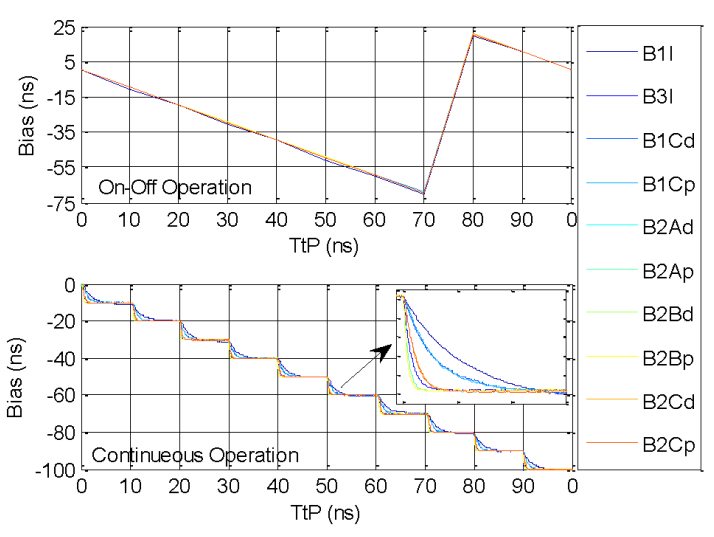

(b)

Figure 11. Ttp impact on channel delay: (a) calibration principle; (b) testing result, with the top panel of on-off operation and the bottom panel of continuous operation.

In the Figure $11 \mathrm{~b}$ the abscissa represented $\mathrm{TtP}$, and the ordinate represents the variation of channel delay. Visibly, under on-off operation, the channel delay represents periodic variation as TtP increased, and there is a fluctuation caused by clock adjustment when $\mathrm{TtP}$ is from $70 \mathrm{~ns}$ to $80 \mathrm{~ns}$. Under continuous operation, the channel delay is monotone decreasing of $10 \mathrm{~ns}$ as $\mathrm{TtP}$ increased by $10 \mathrm{~ns}$. From the watch window in the bottom panel, it can be seen the drift of channel delay for different RF signal is also different. Consequently, the receiver delay is not a fixed value, especially for the receiver having PPS input, which may change a lot along with TtP changing.

\subsubsection{Experiment Verification}

In order to verify the calibration result in the last section, we use two calibrated receivers of UniNav-BDS to test a zero-baseline comparison shown as Figure 12a. The two receivers respectively track satellite RF signal from the same antenna through a power-divider, and rubidium clock of SRS FS725 provides the reference source [26,27]. The $10 \mathrm{MHz}$ reference of the receiver(2) is adjusted by the phase offset generator. Maintaining TtP1 and changing TtP2, we compare the pseudorange bias between two receivers which can directly represent the variation of channel delay [28]. Satellite results of B1I and B3I are selected to verify the calibration result shown as Figure 12b. The earlier abscissa 
is the testing time, and the later abscissa represents $\mathrm{TtP} 2$ of receivers(2), and the ordinate represents pseudorange bias between two receivers.

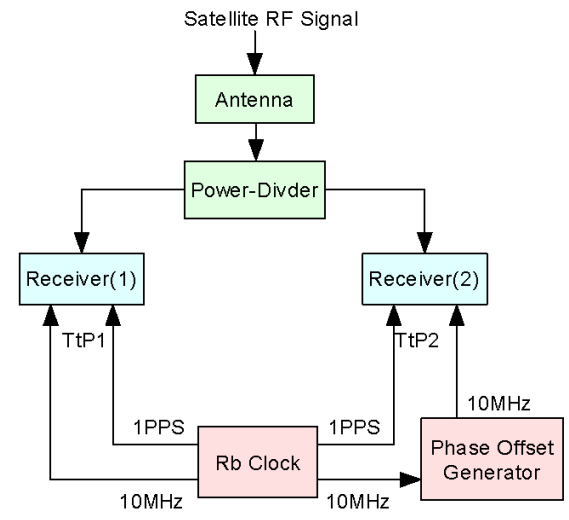

(a)

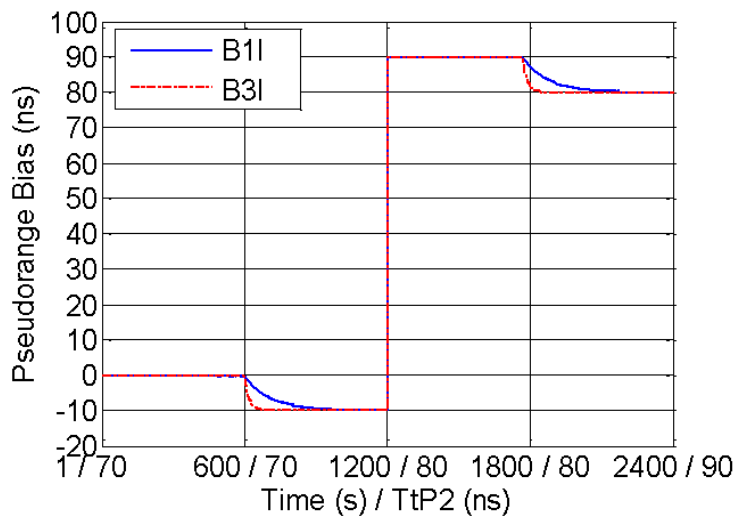

(b)

Figure 12. Zero-baseline comparison: (a) testing principle; (b) testing result.

Step 1: 0-600 s, TtP1 and TtP2 are both kept to $70 \mathrm{~ns}$, and the pseudorange bias of two receivers is better than $0.5 \mathrm{~ns}$.

Step 2: $600-1200 \mathrm{~s}$, under continuous operation, TtP2 increases by $10 \mathrm{~ns}$ namely TtP2 $=80 \mathrm{~ns}$. The pseudorange of the receiver(2) gradually reduces by $10 \mathrm{~ns}$ at first, which tends to be stable during 900-1200 s.

Step 3: 1200-1800 s, under on-off operation, both of two receivers are restarted at $1200 \mathrm{~s}$, and the pseudorange of the receiver(2) jumped by $100 \mathrm{~ns}$. From then on the pseudorange bias of two receivers are better than $0.5 \mathrm{~ns}$ like step 1 .

Step 4: $1800-2400 \mathrm{~s}$, under continuous operation, TtP2 increases by $10 \mathrm{~ns}$ namely TtP2 $=90 \mathrm{~ns}$. The pseudorange of the receiver(2) also gradually reduces by $10 \mathrm{~ns}$ at first, which tends to be stable during 2100-2400 s like step 2.

As a result, the first step represents the calibration consistency of two receivers is better than $0.5 \mathrm{~ns}$. When TtP2 increased, step 2 and step 4 respectively verify the relation between pseudorange and TtP under continuous operation, and step 3 verifies the relation under on-off operation. Therefore, the zero-baseline comparison is consistent with the calibration result between $\mathrm{TtP}$ and channel delay.

\subsection{Analysis on Calibration Uncertainty}

Using the simulator for absolute calibration can eliminate the influence of ephemeris error, clock error, atmosphere error, and multipath interference, which further improves the calibration precision [24,29]. There are still some calibration errors in the experiment, mainly including instrument errors and measurement errors, and it is necessary to analyze the calibration uncertainty, as shown in Table 3 [30].

Table 3. Uncertainty analysis of absolute calibration (unit: ns).

\begin{tabular}{cc}
\hline Error Source & Uncertainty \\
\hline Simulator & 1 \\
Oscilloscope & 0.2 \\
Amplifier, cable, adapter & 0.5 \\
Vector network analyzer & 0.2 \\
Time interval counter & 0.1 \\
Pseudorange & 0.5 \\
Receiver PPS output & 0.5 \\
Combined Uncertainty & $\mathbf{1 . 3 6}$ \\
\hline
\end{tabular}


The simulator are mainly reflected on $\mathrm{TtC}$, like the code phase adjustment precision, $\mathrm{RF}$ signal stability, with uncertainty $1 \mathrm{~ns}$. The oscilloscope is mainly affected by the noise ratio, with uncertainty $0.2 \mathrm{~ns}$. Amplifiers delay measurement by the vector network analyzer, the amplifier, cable and adapter are mainly affected by phase noise and ripple interference, with uncertainty $0.5 \mathrm{~ns}$, and the vector network analyzer is mainly reflected on group delay measurement, with uncertainty $0.2 \mathrm{~ns}$. The time interval counter is mainly affected by the trigger level and channel offset, with uncertainty $0.1 \mathrm{~ns}$. The pseudorange from the receiver is mainly reflected on tracking precision and measurement fluctuation, with uncertainty $0.5 \mathrm{~ns}$. Receiver PPS output is mainly affected by clock stability and adjustment precision, with uncertainty $0.5 \mathrm{~ns}$.

In summary, the combined uncertainty of absolute calibration can be calculated with $1.36 \mathrm{~ns}$ by the standard deviation.

\section{Discussion}

From the calibration results in Section 4.2, the receiver delay of different types maybe differ a lot. Meanwhile, from the calibration results in Section 4.3, the delay of the same receiver under different TtP is also different. GNSS receiver delay is not a fixed value, not only affected by temperature, SNR and aging rate, but also affected by operation model, parameter setting, reference clock, etc. It means the receiver delay may be changed in the outfield although it has just been calibrated in the laboratory, which should be paid much attention.

For the receivers without PPS input, the calibration of channel delay can also deduct clock-bias from receiver pseudorange. However, for the receivers with PPS input, although deducting the clock-bias may nearly eliminate the influence of $\mathrm{TtP}$ on calibration, the receiver system time will steer to GNSST instead of PPS input. Thus the receiver pseudorange cannot represent the measurement based on external PPS in the application. That is why we consider the clock-bias is part of channel delay.

Some researchers may calibrate the receiver together with the antenna in the anechoic chamber [31]. The group delay of the antenna is mainly related to frequency bands, and the inter-frequency bias of the antenna may be much larger than the receiver. In this way, the calibration result will include receiver delay, cable delay, and antenna delay. If we changes the link delay larger such as increasing the cable length, the receiver pseudorange and clock-bias will become larger synchronously. Once the clock-bias exceeds adjustment threshold, the receiver will adjust clock-bias to steer to GNSST/PPS, and the total delay may become smaller on the contrary.

Author Contributions: Conceptualization, F.Z. and H.Z.; methodology, H.Z. and L.H.; validation, X.L. and P.F.; formal analysis, X.L.; investigation F.Z. and H.Z.; data curation, L.H.; writing-original draft preparation, F.Z.; review and editing, X.L.; supervision, X.L. and P.F.; funding acquisition, F.Z.; All authors have read and agreed to the published version of the manuscript.

Funding: The work is funded by the National Natural Science Funds of China (Grant No: 11703032) and Cooperative Precise Positioning Technology of 2016 China National Key Research and Development Plan (Grant No: 2016YFB0501804).

Acknowledgments: The authors would like to thank Longxia $\mathrm{Xu}$, Yinhua Liu, Lin Zhu, and Rui Tu, also with their colleagues for testing the experiment and data provided in the manuscript.

Conflicts of Interest: The authors declare no conflict of interest.

\section{References}

1. Teunissen, P.J.G.; Montenbruck, O. Springer Handbook of Global Navigation Satellite Systems; Springer: Berlin, Germany, 2017.

2. Al-Shaery, A.; Zhang, S.; Rizos, C. An Enhanced Calibration Method of GLONASS Inter-channel Bias for GNSS RTK. GPS Solut. 2013, 17, 165-173. [CrossRef]

3. Costa, R.; Orgiazzi, D.; Pettiti, V.; Sesia, I.; Tavella, P. Performance Comparison and Stability Characterization of Timing and Geodetic GPS Receivers at IEN. In Proceedings of the 18th European Frequency and Time Forum, Guildford, UK, 5-7 April 2004; pp. 279-286. 
4. Levine, J. A review of time and frequency transfer methods. Metrologia 2008, 45, S162-S174. [CrossRef]

5. Guyennon, N.; Cerretto, G.; Tavella, P. Further Characterization of the Time Transfer Capabilities of Precise Point Positioning (PPP). Fish Physiol. Biochem. 2007, 5, 399-404.

6. Defraigne, P.; Petit, G. Time Transfer to TAI Using Geodetic Receivers. Metrologia 2003, 40, 184-188. [CrossRef]

7. Fonville, B.; Powers, E.; Ioannides, R.; Hahn, J.; Mudrak, A. Timing Calibration of a GPS/Galileo Combined Receiver. In Proceedings of the 44th Precise Time and Time Interval Systems and Applications Meeting, Reston, VA, USA, 26-29 November 2012; pp. 167-178.

8. Li, X.; Zhang, H.; Shi, S.; Wang, G. Measurement of the Time Delay of GPS Timing Receiver Based on UTC (NTSC). In Proceedings of the 2009 IEEE International Frequency Control Symposium Joint with the 22nd European Frequency and Time Forum, Besancon, France, 20-24 April 2009; pp. 1073-1075.

9. Proia, A.; Cibiel, G.; White, J.; Wilson, D.; Senior, K. Absolute Calibration of GNSS Time Transfer Systems: NRL and CNES Techniques Comparison. In Proceedings of the Joint Meeting European Frequency and Time Forum and IEEE International Frequency Control Symposium, San Francisco, CA, USA, 2-5 May 2011; pp. 1-6.

10. Grunert, U.; Thoelert, S.; Denks, H.; Furthner, J. Using of spirent GPS/Galileo HW simulator for timing receiver calibration. In Proceedings of the IEEE/ION, Monterey, CA, USA, 5-8 May 2008; pp. 77-81.

11. Petit, G.; Jiang, Z. GPS All in View time transfer for TAI computation. Metrologia 2007, 45, 35-45. [CrossRef]

12. Hegarty, C.; Powers, E.; Fonville, B. Accounting for Timing Biases between GPS, Modernized GPS, and Galileo Signals. In Proceedings of the 36th Annual Precise Time and Time Interval Systems and Applications Meeting, Washington, DC, USA, 7-9 December 2004; pp. 307-317.

13. Landis, G.; White, J. Limitation of GPS Receiver Calibrations. Int. J. Infect. Dis. 2005, 3, 230-236.

14. Pan, L.; Guo, F.; Ma, F. An Improved BDS Satellite-Induced Code Bias Correction Model Considering the Consistency of Multipath Combinations. Remote Sens. 2018, 10, 1189. [CrossRef]

15. Odijk, D.; Teunissen, P.J.G. Characterization of Between-receiver GPS-Galileo Inter-system Biases and Their Effect on Mixed Ambiguity Resolution. GPS Solut. 2013, 17, 521-533. [CrossRef]

16. Wanninger, L. Carrier-phase Inter-frequency Biases of GLONASS Receivers. J. Geod. 2012, 86, 139-148. [CrossRef]

17. Shmaliy, Y.; Ibarra-Manzano, O.; Arceo-Miquel, L. Efficient Predictive Steering of Local Clocks in GPS-based Timekeeping. In Proceedings of the 2009 IEEE International Frequency Control Symposium Joint with the 22nd European Frequency and Time Forum, Besancon, France, 20-24 April 2009; pp. 727-732.

18. Zhu, F.; Li, X.; Zhang, H. Absolute Calibration of Timing Receiver Based on Clock-steering Model. Chin. J. Sci. Instrum. 2014, 35, 1946-1954.

19. Petit, G.; Jiang, Z.; Uhrich, P.; Taris, F. Differential Calibration of Ashtech Z12-T Receivers for Accurate Time Comparisons. In Proceedings of the 14th European Frequency and Time Forum, Torino, Italy, 14-16 March 2000; pp. 40-44.

20. Petit, G.; Jiang, Z.; White, J.; Beard, R.; Powers, E. Absolute Calibration of an Ashtech Z12-T GPS Receiver. GPS Solut. 2001, 4, 41-46. [CrossRef]

21. Zhang, Z.; Li, B.; Shen, Y.; Yang, L. A Noise Analysis Method for GNSS Signals of a Standalone Receiver. Acta Geod. Geophys. 2017, 52, 301-316. [CrossRef]

22. Hauschild, A.; Montenbruck, O. A study on the Dependency of GNSS Pseudorange Biases on Correlator Spacing. GPS Solut. 2016, 20, 159-171. [CrossRef]

23. Zhu, F.; Zhang, H.; Li, X.; Liu, Y. Influence Analysis of the Phase Difference between the Output Reference Signals on Receiver Zero-value. Chin. J. Sci. Instrum. 2015, 36, 1592-1597.

24. Plumb, J.; Larson, K.M.; White, J.; Powers, E. Absolute calibration of a geodetic time transfer system. IEEE Trans. Ultrason. Ferroelectr. Freq. Control. 2005, 52, 1904-1911. [CrossRef] [PubMed]

25. Tu, R.; Zhang, P.; Zhang, R.; Liu, J.; Lu, X. Modeling and Assessment of Precise Time Transfer by Using BeiDou Navigation Satellite System Triple-frequency Signals. Sensors 2018, 18, 1017. [CrossRef] [PubMed]

26. Ciećko, A.; Bakuła, M.; Grunwald, G.; Ćwiklak, J. Examination of Multi-Receiver GPS/EGNOS Positioning with Kalman Filtering and Validation Based on CORS Stations. Sensors 2020, 20, 2732. [CrossRef]

27. Zhang, B.; Teunissen, P.J.G. Characterization of Multi-GNSS Between-receiver Differential Code Biases Using Zero and Short Baselines. Sci. Bull. 2015, 60, 1840-1849. [CrossRef] 
28. Zhong, X.; Tang, Z.; Meng, Z.; Ming, D. A Study on High Precision Calibration of Zero Value for Navigation Signal Simulator. In Proceedings of the China Satellite Navigation Conference, Wuhan, China, 15-17 May 2013; Springer: Berlin/Heidelberg, Germany, 2013; pp. 469-477.

29. Krietemeyer, A.; Van Der Marel, H.; Van De Giesen, N.; Ten Veldhuis, M.C. High Quality Zenith Tropospheric Delay Estimation Using a Low-cost Dual-frequency Receiver and Relative Antenna Calibration. Remote Sens. 2020, 12, 1393. [CrossRef]

30. Zhang, Q.; Niu, X.; Shi, C. Impact Assessment of Various IMU Error Sources on the Relative Accuracy of the GNSS/INS Systems. IEEE Sens. J. 2020, 20, 5026-5038. [CrossRef]

31. Baire, Q.; Pottiaux, E.; Bruyninx, C.; Defraigne, P.; Aerts, W.; Legrand, J.; Bergeot, N.; Chevalier, J.M. Influence of Different GPS Receiver Antenna Calibration Models on Geodetic Positioning. GPS Solut. 2014, 18, 529-539. [CrossRef]

Publisher's Note: MDPI stays neutral with regard to jurisdictional claims in published maps and institutional affiliations.

(C) 2020 by the authors. Licensee MDPI, Basel, Switzerland. This article is an open access article distributed under the terms and conditions of the Creative Commons Attribution (CC BY) license (http://creativecommons.org/licenses/by/4.0/). 